\title{
ASYMPTOTIC EQUIVALENCE OF ALMOST PERIODIC SOLUTIONS FOR A CLASS OF PERTURBED ALMOST PERIODIC SYSTEMS
}

\author{
MANUEL PINTO \\ Departamento de Matemáticas, Facultad de Ciencias, Universidad de Chile, \\ Casilla 653, Santiago 7800024, Chile \\ e-mail:pintoj@uchile.cl \\ VICTOR TORRES \\ Departamento de Ciencias Físicas y Matemáticas, Universidad Arturo Prat, \\ Avda. Arturo Prat 2120, Iquique 1110939, Chile \\ e-mail:vtorres@unap.cl \\ and GONZALO ROBLEDO \\ Departamento de Matemáticas, Facultad de Ciencias, Universidad de Chile, \\ Casilla 653, Santiago 7800024, Chile \\ e-mail: grobledo@uchile.cl
}

(Received 9 June 2009; revised 30 December 2009; accepted 17 June 2010)

\begin{abstract}
The solutions of a perturbed linear ordinary differential equation (ODE) system are studied. Provided that some integrability and oddness conditions are satisfied, we show that they are asymptotically equivalent at $t= \pm \infty$ to the solutions of the unperturbed one. This fact is used to determine the existence of almost periodic or pseudo-almost periodic solutions of the perturbed system.
\end{abstract}

2010 Mathematics Subject Classification. 34E10, 34C27, 34C41.

1. Introduction. In this note, we consider the perturbed systems of ordinary differential equations

$$
\begin{aligned}
& y^{\prime}=A(t) y+f(t, y), \\
& z^{\prime}=A(t) z+B(t) z+h(t)
\end{aligned}
$$

and its relation with the linear system

$$
x^{\prime}=A(t) x .
$$

By following an idea developed by Akhmet et al. [1] for the linear system (1.2), we obtain sufficient conditions ensuring the asymptotic equivalence between (1.1)-(1.2) and (1.3). The results are used to prove the existence of asymptotically almost periodic solutions and pseudo-almost periodic solutions of (1.1)-(1.2) provided that (1.3) has non-trivial almost periodic solutions.

Throughout this note, $f: \mathbb{R} \times \mathbb{R}^{n} \mapsto \mathbb{R}^{n}, h: \mathbb{R} \mapsto \mathbb{R}^{n}$ are continuous. $A(\cdot), B(\cdot)$ are $n \times n$ continuous matrices. A solution of (1.1) with initial condition $y\left(t_{0}\right)=b \in \mathbb{R}^{n}$ will be denoted as $y\left(t, t_{0}, b\right)$, the fundamental matrix of (1.3) is denoted by $\Phi(t)$ and 
$\Phi(t, s)=\Phi(t) \Phi^{-1}(s)$, the matrix and vectorial norm are denoted by $\|\cdot\|$ and $|\cdot|$, the Landau's notation:

$$
p_{1}(t)=o(1) \quad \text { and } \quad p_{1}(t)=O\left(p_{2}(t)\right)
$$

means, respectively, that $\lim _{t \rightarrow+\infty} p_{1}(t)=0$ and that there exist a constant $M$ verifying $\left|p_{1}(t)\right|<M\left|p_{2}(t)\right|$ for big values of $t$. Finally, throughout this note, we assume that

(L) There exists a measurable function $\eta: \mathbb{R} \mapsto \mathbb{R}_{+}$such that

$$
\left|f\left(t, y_{1}\right)-f\left(t, y_{2}\right)\right| \leq \eta(t)\left|y_{1}-y_{2}\right| \quad \text { for any } \quad t \in \mathbb{R} \quad \text { and } \quad y_{1}, y_{2} \in \mathbb{R}^{n} .
$$

Our main results are:

THEOREM 1. If the following conditions are satisfied:

(H1) $t \mapsto\left\|\Phi^{-1}(t)\right\|\|\Phi(t)\| \eta(t)$ and $t \mapsto \Phi^{-1}(t) f(t, 0)$ are integrable on $(0,+\infty)$, (H2) $\int_{t}^{+\infty}\|\Phi(t, s)\|\|\Phi(s)\| \eta(s) d s=o(1)$ and $\lim _{t \rightarrow+\infty} \int_{t}^{+\infty} \Phi(t, s) f(s, 0) d s=0$, then all the solutions of (1.1) are defined on $\mathbb{R}_{+}$and there exists a homeomorphism between the solutions $x(t)=x(t, 0, b)$ of system $(1.3)$ and $y(t)=y(t, 0, \mathcal{H}(b))$ of (1.1), where $\mathcal{H}$ is a $\mathbb{R}^{n}$-homeomorphism and $b$ is arbitrary. Moreover, these solutions satisfy:

$$
\lim _{t \rightarrow+\infty}|x(t)-y(t)|=0
$$

In addition, if the assumptions

(H3) $t \mapsto \Phi^{-1}(t) f(t, \Phi(t) x) i$ odd for any $x \in \mathbb{R}^{n}$,

(H4) $\lim _{t \rightarrow-\infty} \int_{-\infty}^{t}\|\Phi(t, s)\|\|\Phi(s)\| \eta(s) d s=0$ and $\lim _{t \rightarrow-\infty} \int_{-\infty}^{t} \Phi(t, s) f(s, 0) d s=0$ are satisfied, then the solutions satisfy:

$$
\lim _{t \rightarrow-\infty}|x(t)-y(t)|=0 .
$$

THEOREM 2. If the following conditions are satisfied:

(L1) $t \mapsto\left\|\Phi^{-1}(t) B(t) \Phi(t)\right\|$ and $t \mapsto \Phi^{-1}(t) h(t)$ are integrable on $(0,+\infty)$,

(L2) $\int_{t}^{+\infty}\|\Phi(t, s) B(s) \Phi(s)\| d s=o(1)$ and $\lim _{t \rightarrow+\infty} \int_{t}^{+\infty} \Phi(t, s) h(s) d s=0$,

then the solutions of (1.2) are defined on $\mathbb{R}_{+}$and there exists a homeomorphism between the solutions $x(t)=x(t, 0, b)$ of $(1.3)$ and $z(t)=z(t, 0, \mathcal{H}(b))$ of (1.2), where $\mathcal{H}$ is $a$ $\mathbb{R}^{n}$-homeomorphism and $b$ is arbitrary. Moreover, the solutions satisfy

$$
\lim _{t \rightarrow+\infty}|x(t)-z(t)|=0 .
$$

Moreover, if the conditions

(L3) $t \mapsto \Phi^{-1}(t) B(t) \Phi(t)$ and $t \rightarrow \Phi^{-1}(t) h(t)$ are odd .

(L4) $\lim _{t \rightarrow-\infty} \int_{-\infty}^{t}\|\Phi(t, s) B(s) \Phi(s)\| d s=0$ and $\lim _{t \rightarrow-\infty} \int_{-\infty}^{t} \Phi(t, s) h(s) d s=0$, are satisfied, these solutions satisfy $\lim _{t \rightarrow-\infty}|x(t)-z(t)|=0$.

The existence of an homeomorphism between $x(t, 0, b)$ and $y(t, 0, \mathcal{H}(b))$ combined with (1.4) is known as asymptotic equivalence between (1.1) and (1.3) (see [1, 3, 4] and references therein). In addition, a bi-asymptotic equivalence exists when (1.5) is also verified (see [1] for details).

Notice that (L3)-(L4) can be satisfied in several cases. For example, if $\Phi(\cdot)$ is either even or odd and $B(\cdot)$ is odd, then $\Phi^{-1}(t) B(t) \Phi(t)$ is odd. Notice that if $A(\cdot)$ is odd, then $\Phi(\cdot)$ is even. Finally, $\Phi^{-1}(t) B(t) \Phi(t)$ is also odd in other cases: i) when $B(\cdot)$ is odd and 
commutes with $\Phi(\cdot)$. ii) when $A(t)$ commutes with $\int_{0}^{t} A(s) d s$ and $B(t)$. A number of concrete results and examples appear in the last section.

An interesting application of these results is a criterion to determine the existence of pseudo-almost periodic solutions for (1.1)-(1.2). The pseudo-almost periodic functions were introduced by Zhang [22] and generalizes the concept of almost periodic function:

Definition 1. $[\mathbf{1}, \mathbf{7}, \mathbf{1 0}, \mathbf{1 7}, \mathbf{2 1}, \mathbf{2 2}]$ A continuous function $r: \mathbb{R} \mapsto \mathbb{R}^{n}$ is

(i) Almost periodic i.e. $r \in \mathcal{A P}(\mathbb{R})$, if for each $\varepsilon>0$ there exists $\ell_{\varepsilon}>0$ such that every interval of length $\ell_{\varepsilon}$ contains a number $\tau$ with $|r(t+\tau)-r(t)|<\varepsilon$.

(ii) Asymptotically almost periodic i.e. $r \in \mathcal{A} \mathcal{A P}(\mathbb{R})$, if $r=g+\varphi$ with $g \in \mathcal{A} \mathcal{P}(\mathbb{R})$ and $\varphi=o(1)$ as $t \rightarrow+\infty$. In addition, if $\varphi=o(1)$ also for $t \rightarrow-\infty$, we will say that $r \in \mathcal{A} \mathcal{A} \mathcal{P}_{0}(\mathbb{R})$.

(iii) Pseudo-almost periodic i.e. $r \in \mathcal{P} \mathcal{A P}(\mathbb{R})$, if $r=g+\varphi$ with $g \in \mathcal{A P}(\mathbb{R})$ and the ergodic component $\varphi \in \mathcal{P} \mathcal{A} \mathcal{P}_{0}(\mathbb{R})$ is bounded continuous and satisfies

$$
\lim _{\tau \rightarrow+\infty} \frac{1}{2 \tau} \int_{-\tau}^{\tau}|\varphi(t)| d t=0
$$

REMARK 1. Notice that $\mathcal{A P}(\mathbb{R}) \subset \mathcal{A} \mathcal{A} \mathcal{P}_{0}(\mathbb{R}) \subset \mathcal{P} \mathcal{A} \mathcal{P}(\mathbb{R})$ (see e.g. $[\mathbf{1}, \mathbf{2}$, p. 548]).

The existence of $\mathcal{A P}(\mathbb{R}), \mathcal{A} \mathcal{A} \mathcal{P}_{0}(\mathbb{R})$ and $\mathcal{P} \mathcal{A} \mathcal{P}(\mathbb{R})$ solutions is among the most attractive topics in qualitative theory of ordinary differential equations due to their applications, especially in biology $[\mathbf{1 4}, \mathbf{2 0}]$, number theory $[\mathbf{8}, \mathbf{1 1}]$, neural networks $[13,15]$ and physics $[16]$. A well known result relates exponential dichotomy (see e.g. $[6,18])$ with the existence of a unique $\mathcal{A P}(\mathbb{R})$ or $\mathcal{P} \mathcal{A} \mathcal{P}(\mathbb{R})$ solution of perturbed systems:

Proposition 1. [10, Theorem 8.1], [21, Theorem 3.1] Let us consider the perturbed system

$$
y^{\prime}=A(t) y+h(t, y)
$$

where $A(\cdot) \in \mathcal{A P}(\mathbb{R})$ and $h: \mathbb{R} \times \mathbb{R}^{n} \mapsto \mathbb{R}^{n}$ is such that

(i) $h\left(t, y_{0}\right) \in \mathcal{A P}(\mathbb{R})($ resp. $\mathcal{P} \mathcal{A} \mathcal{P}(\mathbb{R}))$ for any $y_{0} \in \mathbb{R}^{n}$.

(ii) There exists $L>0$ such that $|h(t, x)-h(t, y)| \leq L|x-y| \forall x, y \in \mathbb{R}^{n}$.

If the system (1.3) has an exponential dichotomy, then for $L$ sufficiently small it follows that the perturbed system has a unique $\mathcal{A} \mathcal{P}(\mathbb{R})($ resp. $\mathcal{P} \mathcal{A} \mathcal{P}(\mathbb{R}))$ solution.

Without considering exponential dichotomy, the results about existence of $\mathcal{A P}(\mathbb{R})$ solutions are limited in number [10, 12, 22]: Van Vleck [19] and Burton [5] consider $A(\cdot)$ $\omega$-periodic and skew symmetric (i.e. $A(t)=A(t+\omega)$ and $A^{T}(t)=-A(t)$ ) and obtain conditions for the existence of $\mathcal{A P}(\mathbb{R})$ solutions for (1.3). Akhmet et al. [1] recently studied $A(\cdot)$ odd, $B(\cdot)$ even and obtain the existence of $\mathcal{A} \mathcal{A} \mathcal{P}_{0}(\mathbb{R})$ solutions for (1.2), they also define $\mathcal{A} \mathcal{A} \mathcal{P}_{0}(\mathbb{R})$ as bi-asymptotically almost periodic functions.

Our objective is to determine when the non-zero $\mathcal{A P}(\mathbb{R})$ solutions of the linear system (1.3) arise $\mathcal{A P}(\mathbb{R})$ solutions for the perturbed systems (1.1)-(1.2). To work in this framework has two consequences: firstly, exponential dichotomy is not verified because it would imply that zero is the unique $\mathcal{A P}(\mathbb{R})$ solution of (1.3). Secondly, the conditions (i)-(ii) of Proposition 1 are not sufficient to ensure existence of almost periodic solutions for the perturbed systems and new conditions are needed. We will propose a set of integrability and oddness conditions ensuring the existence of a 
manifold of $\mathcal{A} \mathcal{A} \mathcal{P}_{0}(\mathbb{R})$ (resp. $\mathcal{P} \mathcal{A} \mathcal{P}(\mathbb{R})$ ) solutions of (1.1) and (1.2). These results are complementary to the previous ones and are stated as follows:

THEOREM 3. Assume that the system (1.3) has a $k$ parameter $(k \leq n)$ family $\mathcal{S}_{1}$ of $\mathcal{A P}(\mathbb{R})$ (resp. $\mathcal{P} \mathcal{A} \mathcal{P}(\mathbb{R})$ ) solutions. If the assumptions $(\mathbf{H 1})-(\mathbf{H 4})$ are satisfied, then (1.1) has a $k$ parameter family $\mathcal{S}_{2}$ of $\mathcal{A} \mathcal{A} \mathcal{P}_{0}(\mathbb{R})$ (resp. $\mathcal{P} \mathcal{A P}(\mathbb{R})$ ) solutions homeomorphic to $\mathcal{S}_{1}$.

THEOREM 4. Assume that the system (1.3) has a $k$ parameter $(k \leq n)$ family $\mathcal{S}_{1}$ of $\mathcal{A P}(\mathbb{R})$ (resp. $\mathcal{P} \mathcal{A P}(\mathbb{R}))$ solutions. If $B(t)$ and $h(t)$ satisfies the assumptions (L1)(L4) then, (1.2) has a $k$ parameter family $\mathcal{S}_{2}$ of $\mathcal{A} \mathcal{A} \mathcal{P}_{0}(\mathbb{R})$ (resp. $\mathcal{P} \mathcal{A} \mathcal{P}(\mathbb{R})$ ) solutions homeomorphic to $\mathcal{S}_{1}$.

The paper is organized as follows: Section 2 gives a set of preliminary results and the proofs of the Theorems 1 and 2. Theorems 3 and 4 are proved in Section 3, where some illustrative examples and consequences are also included.

2. Existence of solutions and asymptotic equivalence. The following result will be used at several steps of the paper:

THEOREM 5. Let us consider the non-linear system

$$
w^{\prime}=g(t, w)
$$

If $g: \mathbb{R} \times \mathbb{R}^{n} \mapsto \mathbb{R}^{n}$ satisfies the conditions:

(G1) There exists a measurable and integrable function $\xi: \mathbb{R} \mapsto \mathbb{R}_{+}$such that

$$
\left|g\left(t, w_{1}\right)-g\left(t, w_{2}\right)\right| \leq \xi(t)\left|w_{1}-w_{2}\right| \quad \text { for any } \quad t \in \mathbb{R} \quad \text { and } \quad w_{i} \in \mathbb{R}^{n},
$$

(G2) The function $t \mapsto g(t, 0)$ is integrable,

(G3) $g(-t, w)=-g(t, w)$ for any $t \in \mathbb{R}$ and $w \in \mathbb{R}^{n}$,

then there exists a Lipschitz homeomorphism between the solutions of (2.1) and $\mathbb{R}^{n}$ given by the correspondence between $b \in \mathbb{R}^{n}$ and the unique even solution of (2.1) satisfying $\lim _{t \rightarrow \pm \infty} w(t)=b$. More precisely, $w(t)=b-\int_{t}^{+\infty} g(s, 0) d s+\varphi(t)$ with

$$
\varphi(t)= \begin{cases}O\left(\exp \left(\int_{t}^{+\infty} \xi(s) d s\right)-1\right) & t \geq 0 \\ O\left(\exp \left(\int_{-\infty}^{t} \xi(s) d s\right)-1\right) & t<0 .\end{cases}
$$

Proof. Notice that (2.1) can be written as follows:

$$
\dot{w}=\tilde{g}(t, w)+g(t, 0) \quad \text { with } \quad \tilde{g}(t, w)=g(t, w)-g(t, 0) .
$$

Firstly, we will study the solutions defined on $t \geq 0$, the existence is proved by using successive approximations: let $w_{0}(t)=b-\int_{t}^{+\infty} \bar{g}(s, 0) d s$ and

$$
w_{k}(t)=b-\int_{t}^{+\infty} g(s, 0) d s-\int_{t}^{+\infty} \tilde{g}\left(s, w_{k-1}(s)\right) d s \quad k \geq 1 .
$$


By induction and (G1)-(G2), it is straightforward to prove that for $t \geq 0$ :

$$
\left|w_{k}(t)-w_{k-1}(t)\right| \leq \frac{|b|+\rho}{k !}\left(\int_{t}^{\infty} \xi(s) d s\right)^{k}, \quad \rho=\sup _{t \geq 0}\left|\int_{t}^{\infty} g(s, 0) d s\right|,
$$

combining this fact with $w_{k}(t)=w_{0}(t)+\sum_{j=0}^{k-1}\left\{w_{j+1}(t)-w_{j}(t)\right\}$, it follows that

$$
\left|w_{k}(t)\right| \leq|b|+\rho+\sum_{j=1}^{k-1} \frac{|b|+\rho}{j !}\left(\int_{t}^{\infty} \xi(s) d s\right)^{j} .
$$

Assumption (G1) implies that $w_{k}$ is uniformly convergent to a bounded and continuous function $w(t)$. By letting $k \rightarrow \infty$, it follows that

$$
w(t)=b-\int_{t}^{\infty} g(s, w(s)) d s=w_{0}(t)-\int_{t}^{\infty} \tilde{g}(s, w(s)) d s, \quad t \geq 0
$$

and the existence of a solution $w(\cdot)$ convergent toward $b$ when $t \rightarrow+\infty$ follows. Notice that on $\mathbb{R}, t \rightarrow w_{0}(t)$ is even since $t \rightarrow g(t, 0)$ is odd. Moreover, it can be proved by induction that on $\mathbb{R}, t \rightarrow w_{k}(t)$ is even and we conclude that $w(t)$ is even on $\mathbb{R}$.

The asymptotic estimation (2.2) can be proved by letting $k \rightarrow+\infty$ in (2.3) combined with (G1) and (2.4).

Given two solutions $w_{i}(t)(i=1,2)$ satisfying $\lim _{t \rightarrow+\infty} w_{i}(t)=b_{i}$, let us denote $u(t)=w_{1}(t)-w_{2}(t)$. By using (G1) combined with (2.4), we can prove that

$$
|u(t)| \leq\left|b_{1}-b_{2}\right|+\int_{t}^{\infty} \xi(s)|u(s)| d s .
$$

By following Gronwall's Lemma arguments, we can deduce that

$$
\left|w_{1}(t)-w_{2}(t)\right| \leq e^{\int_{t}^{\infty} \xi(s) d s}\left|b_{1}-b_{2}\right|, \quad t \geq 0
$$

and the uniqueness of the solution convergent to $b$ follows.

Moreover, there exists a homeomorphism $\mathcal{H}: \mathbb{R}^{n} \mapsto \mathbb{R}^{n}$ defined by $\mathcal{H}(b)=w(0)$, the initial condition at $t=0$ of the solution $w(t) \rightarrow b$. Indeed, the bijectivity is consequence of the existence and uniqueness of the solutions of (2.1).

Finally, it can be proved by Gronwall's inequality that

$$
\left|w_{1}(t)-w_{2}(t)\right| \leq e^{\int_{0}^{t} \xi(s) d s}\left|w_{1}(0)-w_{2}(0)\right|, \quad t \geq 0
$$

and we can conclude that

$$
e^{-\int_{0}^{\infty} \xi(s) d s}\left|w_{1}(0)-w_{2}(0)\right| \leq\left|b_{1}-b_{2}\right| \leq e^{\int_{0}^{\infty} \xi(s) d s}\left|w_{1}(0)-w_{2}(0)\right| .
$$

By the evenness of $w(\cdot)$, this result is valid on $\mathbb{R}$. Hence, $\mathcal{H}$ is a uniform homeomorphism and the Theorem follows.

COROLlary 1. Let us consider the linear system

$$
w^{\prime}=F(t) w+\gamma(t)
$$

where $F(t)$ is a continuous and integrable matrix function and $\gamma: \mathbb{R} \mapsto \mathbb{R}^{n}$ is integrable. If $F(\cdot)$ and $\gamma(\cdot)$ are odd functions, then for any $b \in \mathbb{R}^{n}$ there exists a unique even solution 
satisfying $\lim _{t \rightarrow \pm \infty} w(t)=b$. More precisely, we have $w(t)=b-\int_{t}^{+\infty} \gamma(s) d s+\varphi(t)$ with

$$
\varphi(t)= \begin{cases}O\left(\exp \left(\int_{t}^{+\infty}\|F(s)\| d s\right)-1\right) & t \geq 0 \\ O\left(\exp \left(\int_{-\infty}^{t}\|F(s)\| d s\right)-1\right) & t<0 .\end{cases}
$$

The Theorem 5 and Corollary 1 will be applied in order to prove the Theorems 1 and 2.

2.1. Proof of Theorem 1. Let $y(t)$ be a solution of system (1.1) and $u(t)=$ $\Phi^{-1}(t) y(t)$. It is straightforward to prove that system (1.1) becomes

$$
u^{\prime}=\Phi^{-1}(t) f(t, \Phi(t) u)=g(t, u)
$$

and the conditions (G1)-(G2) of Theorem 1 can be verified by using (L) and (H1).

Firstly, let us consider $t \geq 0$. By Theorem 5 , for any $b \in \mathbb{R}^{n}$ there exists a unique solution $u(t)$ convergent to $b$ when $t \rightarrow+\infty$. Furthermore, there is a homeomorphism $\mathcal{H}: \mathbb{R}^{n} \mapsto \mathbb{R}^{n}$ such that $\mathcal{H}(b)=u(0)=y(0)$ and $\lim _{t \rightarrow+\infty} \Phi^{-1}(t) y(t)=\lim _{t \rightarrow+\infty} u(t)=b$.

For any $b \in \mathbb{R}$, we consider a solution $y(t, 0, \mathcal{H}(b))$ of $(1.1)$. By Theorem 3 applied to (2.5), there exists a function $y(t)$ solving the integral equation

$$
y(t)=\Phi(t, 0) b-\int_{t}^{+\infty} \Phi(t, s) f(s, \Phi(s) u(s)) d s, \quad t \geq 0,
$$

where $u(t)=u(t, 0, \mathcal{H}(b))$ is solution of (2.5). It is clear that a given initial condition $\mathcal{H}(b)$ results in a homeomorphism between $x(t, 0, b)=\Phi(t, 0) b$ and $y(t)$. Moreover, notice that

$$
|y(t)-x(t)| \leq \int_{t}^{\infty}\|\Phi(t, s)\|\|\Phi(s)\||u(s)| \eta(s) d s+\left|\int_{t}^{\infty} \Phi(t, s) f(s, 0) d s\right| .
$$

By (H2), we can see that (1.4) follows. Moreover, (H3) implies that (2.6) holds for $t \leq 0$ since (2.5) satisfies (G3) and Theorem 5 follows. Finally, (1.5) is a consequence of (H4) and the Theorem is proved.

2.2. Proof of Theorem 2. Notice that $u(t)=\Phi^{-1}(t) z(t)$ transforms (1.2) in

$$
u^{\prime}=\Phi^{-1}(t) B(t) \Phi(t) u+\Phi^{-1}(t) h(t) .
$$

As (L1)-(L3) implies that (2.7) satisfies assumptions of Corollary 1. Following the lines of the previous proof, we obtain a representation $y(t)=x(t)+\varphi(t)$ with

$$
\varphi(t)=-\int_{t}^{+\infty} \Phi(t, s) B(s) \Phi(s) u(s) d s-\int_{t}^{+\infty} \Phi(t, s) h(s) d s \quad t \geq 0
$$

and (L2) combined with (L4) imply $\lim _{t \rightarrow \pm \infty}|x(t)-z(t)|=0$. 


\section{Existence of $\mathcal{P} \mathcal{A P}(\mathbb{R})$ solutions and examples.}

3.1. Proofs of Theorems 3 and 4. The proofs follow immediately from Theorems 1 and 2 respectively. We will prove only Theorem 3: let $x(t)=x(t, 0, b) \in \mathcal{S}_{1}$. By Theorem 1, there exists a solution of (1.1) described by $y(t)=y(t, 0, \mathcal{H}(b))=x(t)+$ $\varphi(t)$, where $\varphi$ is given by

$$
\varphi(t)=-\Phi(t) \int_{t}^{+\infty} \Phi^{-1}(s) f(s, y(s)) d s
$$

such that $\lim _{t \rightarrow \pm \infty} \varphi(t)=0$. Using this fact combined with $x(\cdot) \in \mathcal{A P}(\mathbb{R})$ (resp. $x(\cdot) \in \mathcal{P} \mathcal{A} \mathcal{P}(\mathbb{R}))$ implies that $y(\cdot) \in \mathcal{A} \mathcal{A} \mathcal{P}_{0}(\mathbb{R})($ resp. $y(\cdot) \in \mathcal{P} \mathcal{A} \mathcal{P}(\mathbb{R}))$ and the Theorem 1 follows.

\subsection{Consequences and illustrative examples.}

EXAMPLE 1. Assume that (H1)-(H4) are satisfied. If $A(\cdot)$ is $\omega$-periodic and skew symmetric (i.e. $A(t)=A(t+\omega)$ and $\left.A^{T}(t)=-A(t)\right)$, then all the solutions of (1.1) are $\mathcal{A} \mathcal{A} \mathcal{P}_{0}(\mathbb{R})$ solution. Indeed, Theorem 1 from [5] says that all the solutions of (1.3) are $\mathcal{A P}(\mathbb{R})$ and the result follows from Theorem 3 .

THEOREM 6. Let $\mathcal{S}_{1}$ be a $k$ parameter family of $\mathcal{A P}(\mathbb{R})$ solutions of (1.3):

(i) If (H1)-(H2) are satisfied, then there exists a $k$ parameter family $\mathcal{S}_{2}$ of $\mathcal{A} \mathcal{A P}(\mathbb{R})$ solutions of (1.1) homeomorphic to $\mathcal{S}_{1}$.

(ii) Moreover, if $t \mapsto A(t)$ and $t \mapsto f(t, x)$ are odd for any $x \in \mathbb{R}$, then there exists a $k$ parameter family $\mathcal{S}_{2}$ of $\mathcal{A} \mathcal{A} \mathcal{P}_{0}(\mathbb{R})$ solutions of $(1.1)$ homeomorphic to $\mathcal{S}_{1}$.

Proof. (i) is a straightforward consequence of Theorems 1 and 3. Now we prove (ii): notice that $\Phi(\cdot)$ is even since $A(\cdot)$ is odd and we can conclude that (H3) is satisfied. Following the lines of the proof of Theorem 1 combined with Theorem 5, we can prove that $\varphi(t)$ is an even function. Finally, the asymptotic behaviour (1.4) is obtained by letting $t \rightarrow \pm \infty$ in (2.6) and using (H2) combined with the evenness of $\varphi(t)$.

Similarly,

THEOREM 7. Let $\mathcal{S}_{1}$ be a $k$ parameter family of $\mathcal{P} \mathcal{A} \mathcal{P}(\mathbb{R})$ solutions of (1.3):

(i) If (H1)-(H2) are satisfied, then there exists a $k$ parameter family $\mathcal{S}_{2}$ of $\mathcal{A} \mathcal{A P}(\mathbb{R})$ solutions of (1.1) homeomorphic to $\mathcal{S}_{1}$.

(ii) Moreover, if $t \mapsto A(t)$ and $t \mapsto f(t, x)$ are odd for any $x \in \mathbb{R}$, then there exists a $k$ parameter family $\mathcal{S}_{2}$ of $\mathcal{P} \mathcal{A P}(\mathbb{R})$ solutions of (1.1) homeomorphic to $\mathcal{S}_{1}$.

THEOREM 8. If (G1)-(G2) are satisfied, then every solution of (2.1) is $\mathcal{A} \mathcal{A P}(\mathbb{R})$. In addition, if (G3) holds, then every solution is $\mathcal{A} \mathcal{A} \mathcal{P}_{0}(\mathbb{R})$.

EXAMPLE 2. If (H1)-(H2) are satisfied, $t \mapsto f(t, x)$ is odd for any $x \in \mathbb{R}^{n}$ and $A(t)$ is odd and $\omega$-periodic, then all solutions of (1.1) are $\mathcal{A} \mathcal{A} \mathcal{P}_{0}(\mathbb{R})$ : Indeed, Theorem 2 from [9] says that all the solutions of (1.3) are $\omega$-periodic and the result follows from Theorem 6.

Observe that in this last example, we are in presence of a bi-asymptotically periodic equivalence. In addition, all the solutions of examples 1 and 2 are $\mathcal{A} \mathcal{A P}(\mathbb{R})$ or $\mathcal{P} \mathcal{A P}(\mathbb{R})$. 
COROLlARY 2. If (H1)-(H4) (resp. (L1)-(L4)) are satisfied and moreover

(i) $A(t)$ is $\omega$-periodic,

(ii) The linear system (1.3) has a unique bounded solution, then the systems (1.1) (resp.(1.2)) have a solution $\mathcal{A} \mathcal{A} \mathcal{P}_{0}(\mathbb{R})$.

Proof. Let $x(t)$ be the bounded solution of system (1.3) and let $x_{1}(t)=x(t+\omega)$. Notice that $x_{1}(t)$ is also a bounded solution of (1.3). Hence $x_{1}(t)=x(t)$ and $x(t)$ is $\omega$-periodic. The rest of the proof follows from Theorem 1.

COROllary 3. Assume that

(i) $\Phi(t)$ and $B(t)$ commute,

(ii) $B(\cdot)$ is odd and $t \mapsto B(t)$ is integrable,

(iii) $\lim _{t \rightarrow \pm \infty} \Phi(t) \int_{t}^{\infty} B(s) d s=0$

then the conclusions of Theorem 2 holds for $z^{\prime}=A(t) z+B(t) z$.

Corollary 4. Assume that

(i) $A(\cdot)$ and $B(\cdot)$ are odd,

(ii) $t \mapsto \Phi^{-1}(t) B(t) \Phi(t)$ is integrable,

(iii) $\lim _{t \rightarrow+\infty} \int_{t}^{\infty} \Phi(t, s) B(s) \Phi(s) d s=0$

then the conclusions of Theorem 2 holds for $z^{\prime}=A(t) z+B(t) z$.

Observe that Corollary 4 can be compared with Theorem 4.1 from [1] in several ways: firstly, we work with an odd perturbation while in [1] an even perturbation is considered. The integrability condition (ii) is similar. Finally, our condition (iii) is easier to verify in comparison with [1] (See its assumption [C2]).

While Ráb Lemma plays a key role in the asymptotic equivalence results from [1], in our case Theorem 5 makes possible to consider a non-linear version of Corollary 4.1 from [1], as done in Theorems 6 and 7. In addition, this last one extend the results to a $\mathcal{P} \mathcal{A} \mathcal{P}(\mathbb{R})$ case.

COROLlaRY 5. If $t \mapsto f(t, 0)$ and $t \mapsto \eta(t)$ are integrable, (H3) follows, the solutions of (1.3) are bounded and

$$
\liminf _{t \rightarrow+\infty} \int_{0}^{t} \operatorname{Tr} A(s) d s>-\infty
$$

then, from each $k$ parameter family of $\mathcal{A P}(\mathbb{R})$ (resp. $\mathcal{P} \mathcal{A} \mathcal{P}(\mathbb{R})$ ) solutions of $(1.3)$ we obtain an homeomorphic $k$ parameter family of $\mathcal{P} \mathcal{A P}(\mathbb{R})$ solutions of the system (1.1) such that (1.4) follows. A corresponding result holds for system (1.2).

Proof. As $\|\Phi(t)\|$ is bounded, (3.2) implies that $\left\|\Phi^{-1}(t)\right\|$ is bounded. The result follows since the integrability of $\eta(t)$ and $f(t, 0)$ implies (H1), (H2) and (H4).

EXAMPLE 3 . Let $A$ be a $2 n \times 2 n$ matrix with simple purely imaginary eigenvalues $\lambda= \pm i \omega_{k}(k=1, \ldots, n), B(t)$ is a $\mathbb{R}^{2 n} \times \mathbb{R}^{2 n}$ matrix function, such that $A$ commutes with $B(t)$ and $t \mapsto B(t)$ is odd and integrable. Consider the system:

$$
y^{\prime}=A y+B(t) y,
$$

where $y \in \mathbb{R}^{2 n}$, then Corollary 5 implies that any solution of $(3.3)$ is $\mathcal{A} \mathcal{A} \mathcal{P}_{0}(\mathbb{R})$.

Notice that the classic results cannot be applied to this example (see e.g. $[\mathbf{1 0}, \mathbf{2 1}])$ and (1.3) has not an exponential dichotomy. 
EXAMPLE 4. Let us consider the scalar system

$$
y^{\prime}=\{\cos (t)+\cos (\pi t)\} y+t e^{-t^{2}} y .
$$

Here $A(t)$ is even and the solutions $x(t)=e^{\sin (t)+\frac{1}{\pi} \sin (\pi t)} x(0)$ of $(1.3)$ are $\mathcal{A P}(\mathbb{R})$ but neither even nor odd. However, by Corollary 5, every solution $y(\cdot)$ is $\mathcal{P} \mathcal{A P}(\mathbb{R})$. Note that this result cannot be obtained from [1].

4. Discussion. We extend some results and furnish alternative methods compared with [1]: Firstly, we give an alternative proof of asymptotic equivalence that employs Theorem 5 and can be applied also for non-linear systems. On the other hand, we point out that (G1) could be relaxed by supposing that the inequality is verified only locally (we are preparing this result in a future article). Secondly, we can extend the methods of [1] to prove the existence of $\mathcal{P} \mathcal{A} \mathcal{P}(\mathbb{R})$ solutions. Finally, we extend some results from [1] by supposing $B(\cdot)$ odd.

We have given only sufficient conditions for the existence of even $\mathcal{P} \mathcal{A P}(\mathbb{R})$ solutions (resp. $\left.\mathcal{A} \mathcal{A} \mathcal{P}_{0}(\mathbb{R})\right)$. In this sense, a natural extension of our results would be to find sufficient conditions ensuring the existence of solutions that are not even (Example 4 shows us that these solutions exist). Nevertheless, to prove the bi-asymptotic equivalence by using conditions weaker than (G3) remain an open question, worth further study.

ACKNOWLEDGEMENT. The first two authors were supported by FONDECYT 1080034, DGI MATH UNAP 2008. The third author was supported by the Israel Council for Higher Education and UNESCO.

\section{REFERENCES}

1. M. U. Akhmet, M. A. Tleubergenova and A. Zafer, Asymptotic equivalence of differential equations and asymptotically almost periodic solutions, Nonlinear Anal. 67 (2007), $1870-1877$.

2. A. Alonso, J. Hong and R. Obaya, Almost periodic type solutions of differential equations with piecewise constant argument via almost periodic sequences, Appl. Math. Lett. 13 (2000), 131-137.

3. S. Bodine and D. A. Lutz, On asymptotic equivalence of perturbed linear systems of differential and difference equations, J. Math. Anal. Appl. 326 (2007), 1174-1189.

4. F. Brauer and J. S. W. Wong, On asymptotic behavior of perturbed linear systems, J. Differ. Equ. 6 (1969), 142-153.

5. T. A. Burton, Linear differential equations with periodic coefficients, Proc. Amer. Math. Soc. 17 (1966), 327-329.

6. W. A. Coppel. Dichotomies in stability theory. Lecture Notes in Mathematics Vol. 629 (Springer-Verlag, Berlin, New York, 1978).

7. C. Cuevas and M. Pinto, Existence and uniqueness of pseudo almost periodic solutions of semilinear Cauchy problems with non dense domain, Nonlinear Anal. 45 (2001), 73-83.

8. J. Delsarte, Essai sur l'application de la théorie des fonctions presque périodiques à l'arithmétique, Ann. Sci. l'École Normale Supérieure Ser. 3, 62 (1945), 185-204.

9. I. J. Epstein, Periodic solutions of systems of differential equations, Proc. Amer. Math. Soc. 13 (1962), 690-694.

10. A. M. Fink, Almost periodic differential equations. Lecture Notes in Mathematics (Springer-Verlag, Berlin, New York, 1974). 
11. F. Gramain, Fonctions presque périodiques (au sens de Bohr) et fonction zéta de Riemann. Séminaire Delange-Pisot-Poitou. Théorie des nombres, 14 no. 2 (1972-1973), Exposé G17. 6p.

12. Z. S. Hu and A. B. Mingarelli, On a question in the theory of almost periodic differential equations, Proc. Amer. Math. Soc. 127 (1999), 2665-2670.

13. H. Jiang, L. Zhang and Z. Teng, Existence and global exponential stability of almost periodic solution for cellular neural networks with variable coefficients and time varying delays, IEEE Trans. Neural Netw. 16 (2005), 1340-1351.

14. P. Lenas and S. Pavlou, Periodic, quasi-periodic and chaotic coexistence of two competing microbial populations in a periodically operated chemostat, Math. Biosci. 121 (1994), $61-110$.

15. B. Liu and L. Huang, Existence and exponential stability of almost periodic solutions for cellular neural networks with continuously distributed delays, J. Korean Math. Soc. 43 (2006), $445-459$.

16. A. A. Pankov, Bounded and almost periodic solutions of nonlinear operator differential Equations, Mathematics and its applications 55 (Kluwer Academic Publishers, Dordrecht, 1990).

17. D. Piao, Pseudo almost periodic solutions for differential equations involving reflection of the argument, J. Korean Math. Soc. 41 (2004), 747-754.

18. M. Pinto, Pseudo almost periodic solutions of neutral integral and differential equations with applications, Nonlinear Anal. Theory Methods Appl. 72 (2010), 4377-4383.

19. F. S. Van Vleck, A note on the relation between periodic and orthogonal fundamental solutions of linear systems II, Amer. Math. Mon. 71 (1964), 774-776.

20. G. S. K. Wolkowicz and X. Q. Zhao, $N$-specie competition in a periodic chemostat, Differ. Integ. Equ. 11 (1998), 465-491.

21. C. Zhang, Pseudo almost periodic solutions of some differential equations II, J. Math. Anal. Appl. 192 (1995), 543-561.

22. C. Zhang, Almost periodic type functions and ergodicity (Science Press, Beijing; Kluwer Academic Publishers, Dordrecht, 2003). 